\title{
Nota Técnica: \\ Utilización del 'filler' y de superplastificantes en el hormigón de altas prestaciones
}

\author{
Technical Note: \\ Filler and superplasticizer usage on high strength concrete
}

\author{
$\underline{\text { M. Sümer }}{ }^{(*)}$ \\ Recepción/Received: 9-IX-05 \\ Aceptación/Accepted: 20-III-07
}

RESUMEN

En el presente trabajo se estudia la influencia de la utilización de un "filler" (polvo mineral) en el comportamiento del hormigón de altas prestaciones. Para ello, se realizan ensayos de laboratorio en los que se emplean tres series de hormigón, cada una con una dosificación de cemento distinta, de 375,400 y $425 \mathrm{~kg} / \mathrm{m}^{3}$. Se preparan tres grupos de probetas de cada serie, el primero o de referencia con $0 \%$ de aditivo químico y $0 \%$ de "filler", el segundo con un $1,5 \%$ del aditivo químico y $0 \%$ de "filler" y el tercero con un $1,5 \%$ del aditivo químico y un $5 \%$ de "filler" en peso del cemento. Como aditivo se utiliza un superplastificante de nombre comercial "DARACEM 190", siendo el cemento, suministrado por la Nuh Cement Plant, de tipo Portland con una resistencia a la compresión característica de $42,5 \mathrm{~N} / \mathrm{mm}^{2}$. En todas las amasadas se realizan los ensayos del cono de Abrams y de densidad. Para cada serie y grupo, se fabrican dos probetas cúbicas de $15 \times 15 \times 15 \mathrm{~cm}$ que se someten a ensayos de resistencia a la compresión, previo curado en agua a $20 \pm 2^{\circ} \mathrm{C}$ durante $3,7,28$ y 60 días. En total, se fabrican 72 probetas. Los resultados confirman que la utilización del "filler" reduce la porosidad del hormigón, aumentando tanto su densidad como su demanda de agua y mejorando sus propiedades mecánicas.

Palabras clave: puzolanas, resistencia a la compresión, "filler", superplastificantes, altas prestaciones.

\section{SUMMARY}

In this research, the effects of filler (rock-dust) usage on high strength concrete have been investigated through lab experiments and some results have been obtained. The experiments involved three series of concrete with different cement proportions of $375 \mathrm{~kg} / \mathrm{m}^{3}, 400 \mathrm{~kg} / \mathrm{m}^{3}$, and $425 \mathrm{~kg} / \mathrm{m}^{3}$. For each series of concrete, three different groups of samples have been prepared, the first one being the reference concrete which contained $0 \%$ chemical admixture and 0\% filler, the second one contained $1.5 \%$ chemical admixture and $0 \%$ filler and finally the last group contained $1.5 \%$ chemical admixture and $5 \%$ filler to the weight of cement used. The chemical admixture used was a type of Super plasticizer with a brand name of "DARACEM 190", and the cement used was Ordinary Portland Cement of target compressive strength 42.5 $\mathrm{N} / \mathrm{mm}^{2}$, obtained from Nuh Cement Plant. For each batch, Slump Tests and Unit Weight Tests were performed. For each stage and group, two $15 \mathrm{~cm}$ cubic samples have been tested for Compressive Strength after being cured in water at $20 \pm 2{ }^{\circ} \mathrm{C}$ for ages of 3 days, 7 days, 28 and 60 days. The total number of samples was 72. As a result, filler usage was found to reduce the porosity of Concrete, increase the Unit Weight of Concrete, increase the need for water and improve the Compressive Strength Properties of Concrete.

Keywords: puzzolan, compressive strength, filler, superplasticizer, high performances.

(*) Universidad de Sakarya (Sakarya, Turquía).

Persona de contacto/Corresponding author: msumer@sakarya.edu.tr 


\section{INTRODUCCIÓN}

El hormigón es un material de construcción que se compone de áridos, cemento, agua y aditivos químicos y/o minerales (según necesidad), mezclados para formar un producto homogéneo. En los últimos años residuos industriales como la ceniza volante, el humo de silicio, las escorias y el "filler", además de incorporarse en el cemento como adiciones, se han venido añadiendo directamente al hormigón como adiciones. Además, los residuos que se usan directamente con el hormigón han desempeñado un papel muy importante en la generalización del empleo del hormigón preparado. La producción anual de las plantas de este tipo de hormigón ya ha alcanzado niveles superiores a los $10.000 .00 \mathrm{~m}^{3}$. En los últimos años el sector del hormigón ha conseguido utilizar adiciones minerales en las condiciones económicas y tecnológicas propias de empresas de mayor tamaño (1-2).

Uno de los mayores problemas con el que se encuentran los investigadores que trabajan en el campo del hormigón es la determinación de la proporción adecuada de finos que pasan el tamiz número $100(<0,149 \mathrm{~mm})$. Dichos materiales se componen principalmente de arcillas, limos y polvo mineral. La presencia de materiales arcillosos entre los áridos debe evitarse por el efecto negativo que provoca en la adherencia entre éstos y el mortero de cemento, ralentizando la hidratación y aumentando la retracción del hormigón, lo que provoca una pérdida de durabilidad y de resistencia. Con los limos y el polvo mineral ("filler"), en cambio, no ocurre lo mismo.

Los filtros de las canteras donde se producen las arenas y el árido de machaqueo recogen el "filler" (polvo calizo) para no contaminar la atmósfera de las zonas rurales en las que se ubican. El efecto de estos materiales sobre las propiedades del hormigón endurecido puede ser positivo o negativo. Hasta muy recientemente, se ha evitado su utilización, ya que su mayor superficie (consecuencia de su extrema finura) aumenta la relación agua/cemento, ocasionando la retracción del hormigón. Sin embargo, su eliminación del proceso eleva el coste del cemento y reduce su resistencia (3).

Sobre todo en el caso de roca blanda, el machacado genera cantidades ingentes de polvo mineral. Si este material no es de naturaleza arcillosa, podría aplicarse para reducir la porosidad del hormigón, mejorando otras de sus propiedades. Por otra parte, su presencia aumentaría la trabajabilidad del hormigón fabricado con árido de machaqueo. Si el hormigón fresco no contiene una proporción suficiente de finos, limos o polvo mineral, y tras su colocación y compactación no consigue retener el agua de amasado, puede llegar a segregarse. La exudación resultante ocasiona la fisuración del material (4-6).
En estas condiciones, el hormigón adquiere algunas propiedades indeseadas: su porosidad y permeabilidad son altas, su durabilidad reducida y su resistencia a la compresión baja. A fin de evitar estos efectos, se ha de modificar la dosificación del cemento o incorporar aditivos al hormigón, soluciones ambas que implican un sobrecoste. Una manera más adecuada de resolver este problema pasaría por aprovechar el polvo mineral, que no influye en la adherencia entre la matriz y el árido, sino que actúa como "filler". Conviene emplear este tipo de polvo mineral con un superplastificante a fin de mejorar su trabajabilidad y reducir la exudación y con ella la fisuración por retracción. Por todo lo expuesto, se ha procedido a un experimento de laboratorio para determinar la influencia de la presencia del "filler" en las propiedades del hormigón.

\section{ESTUDIO EXPERIMENTAL}

\subsection{Objeto del trabajo y programa experimental}

El objeto del trabajo ha sido el de investigar la influencia del "filler" y del superplastificante en ciertas propiedades del hormigón, y más concretamente en la resistencia a compresión y la durabilidad. Para ello, se han realizado las pruebas con tres dosificaciones de cemento distintas: 375,400 y $425 \mathrm{~kg} / \mathrm{m}^{3}$. Se fabricaron tres amasadas en cada serie: una de referencia sin aditivo, una con sólo el aditivo químico y una con el aditivo químico más el "filler". La dosis del aditivo químico utilizado, un superplastificante de nombre comercial Daracem - 190, fue de $1,5 \%$ en peso del cemento, siendo la del "filler" (polvo mineral) del $5 \%$ en peso del cemento. Por tanto, en este trabajo se utilizaron tres muestras de hormigón de referencia, tres de hormigón con el aditivo químico sólo y tres de hormigón con el aditivo químico más el "filler", por un total de nueve series. De cada una de las series se fabricaron ocho probetas cúbicas de $15 \times 15 \times 15 \mathrm{~cm}$ que, una vez fraguadas, se sumergieron en agua para su curado a una temperatura de $20 \pm 2{ }^{\circ} \mathrm{C}$ durante $3,7,28$ y 60 días.

\subsection{Materiales, proveedores y equipos}

Para los ensayos se utilizó cemento Portland PC 42.5. El árido grueso y el "filler" provenían de la planta de Kartal Settas, y los finos, de la empresa Akpinar. El aditivo químico era el superplastificante Daracem 190. Las amasadas, que eran de $30 \mathrm{I}$, se mezclaron en una amasadora de laboratorio de tipo artesa, con una capacidad de $60 \mathrm{I}$. El agua se agregó paulatinamente hasta alcanzar un asiento de $17 \pm 1 \mathrm{~cm}$ en el cono de Abrams y a continuación se midieron la densidad, el contenido de aire y la temperatura del hormigón. Al llegar a la edad de 3, 7, 28 y 60 días, las probetas (dos por cada edad) se sometieron al ensayo de resistencia 
a la compresión en una prensa de capacidad máxima de 200 toneladas. Las propiedades químicas y físicas del cemento y del "filler" quedan reflejadas en la Tabla
1. En la Tabla 2 se muestran las propiedades físicas del árido y en la Tabla 3, su granulometría y la dosificación de las amasadas.

Tabla 1. Propiedades químicas y físicas del cemento y del "filler"

\begin{tabular}{|c|c|c|}
\hline Composición química (\%) & PC 42.5 & "FILLER" \\
\hline $\mathrm{SiO}_{2}$ (total) & 22,20 & 8,3 \\
\hline $\mathrm{Fe}_{2} \mathrm{O}_{3}$ & 4,39 & 1,3 \\
\hline $\mathrm{Al}_{2} \mathrm{O}_{3}$ & 3,58 & 2,0 \\
\hline $\mathrm{CaO}$ & 63,78 & 46 \\
\hline Free $\mathrm{CaO}$ & 0,82 & - \\
\hline $\mathrm{MgO}$ & 2,06 & 2,42 \\
\hline $\mathrm{SO}_{3}$ & 2,20 & 0,48 \\
\hline Pérdida por calcinación & 1,11 & 38,35 \\
\hline Sólidos insolubles & 0,51 & - \\
\hline $\mathrm{K}_{2} \mathrm{O}+\mathrm{Na}_{2} \mathrm{O}$ & 0,30 & 0,62 \\
\hline \multicolumn{3}{|l|}{ Propiedades físicas } \\
\hline Densidad $\left(\mathrm{g} / \mathrm{cm}^{3}\right)$ & 3,17 & 2,74 \\
\hline Superficie específica $\left(\mathrm{g} / \mathrm{cm}^{2}\right)$ & 3.214 & 3.750 \\
\hline$\%$ Retenido en el tamiz de 200 mm & - & 0,8 \\
\hline$\%$ Retenido en el tamiz de 90 mm & 0,3 & 5,02 \\
\hline$\%$ Retenido en el tamiz de $40 \mathrm{~mm}$ & 14 & 16,04 \\
\hline Humedad (\%) & - & 0,63 \\
\hline Inicio de fraguado (horas:minutos) & $3: 15$ & - \\
\hline Fin de fraguado (horas:minutos) & $4: 00$ & - \\
\hline
\end{tabular}

Tabla 2. Propiedades físicas del árido

\begin{tabular}{|l|c|c|c|c|}
\hline \multicolumn{1}{|c|}{ Materiales } & Arena & Arena de & Árido no I & Árido no II \\
machaqueo & & 2,69 & 2,71 & 2,72 \\
\hline Densidad $\left(\mathrm{g} / \mathrm{cm}^{3}\right)$ & 2,63 & 1,2 & 0,9 & 0,8 \\
\hline Absorción de agua $(\%)$ & 1,1 & 6,8 & - & - \\
\hline$\%$ que pasa el tamiz de $200 \mu \mathrm{m}$ & 2,3 & - & - & - \\
\hline Materia orgánica & - & 1.580 & 1.420 & 1.397 \\
\hline Peso libre por unidad de volumen $\left(\mathrm{kg} / \mathrm{cm}^{3}\right)$ & 1.410 & 1.734 & 1.527 & 1.502 \\
\hline Densidad del hormigón compactado $\left(\mathrm{kg} / \mathrm{cm}^{3}\right)$ & 1.579 & & \\
\hline
\end{tabular}

Tabla 3. Dosificación de las mezclas y granulometría del árido

\begin{tabular}{|c|c|c|c|c|c|}
\hline \multirow[b]{2}{*}{$\begin{array}{l}\text { Tamiz } \\
(\mathrm{mm})\end{array}$} & \multicolumn{5}{|c|}{$\%$ del material que pasa el tamiz } \\
\hline & $\begin{array}{c}\text { Arena } \\
(0-2 \mathrm{~mm})\end{array}$ & $\begin{array}{l}\text { Arena de } \\
\text { machaqueo } \\
(0-5 \mathrm{~mm})\end{array}$ & $\begin{array}{l}\text { Árido } \mathrm{n}^{\circ} \mathrm{I} \\
(5-12 \mathrm{~mm})\end{array}$ & $\begin{array}{l}\text { Árido no II } \\
(12-20 \mathrm{~mm})\end{array}$ & $\begin{array}{c}\text { Granulometría } \\
\text { de la mezcla }\end{array}$ \\
\hline 31,5 & 100 & 100 & 100 & 100 & 100 \\
\hline 16 & 100 & 100 & 100 & 67 & 91 \\
\hline 8 & 100 & 100 & 97 & 4 & 69 \\
\hline 4 & 100 & 83 & 41 & 1 & 52 \\
\hline 2 & 100 & 44 & 21 & 0 & 41 \\
\hline 1 & 100 & 21 & 12 & 0 & 35 \\
\hline 0,5 & 60 & 9 & 8 & 0 & 29 \\
\hline 0,25 & 6 & 3 & 5 & 0 & 4 \\
\hline$\%$ Mezcla & 29 & 11 & 32 & 28 & 100 \\
\hline
\end{tabular}




\subsection{Resultados de las pruebas}

Los resultados de los ensayos de laboratorio se resumen en la Tabla 4. La Figura 1 muestra la evolución de la densidad de las muestras del hormigón fresco y en las Figuras 2 y 3 se representa la resistencia a compresión de las probetas a los 28 y 60 días, respectivamente. La resistencia a compresión de las probetas de hormigón preparado con dosis de cemento de 375,400 y 425 se visualiza en las Figuras 4, 5 y 6, respectivamente.

\section{DISCUSIÓN DE LOS RESULTADOS EXPERIMENTALES}

Este trabajo tenía como objeto el de investigar el efecto de la incorporación de "filler" en algunas de las propiedades del hormigón de altas prestaciones. Los resultados obtenidos han sido los siguientes:
A los 28 días, la resistencia a la compresión del hormigón al que se había incorporado un aditivo químico era de un 25 a un $30 \%$ mayor que la del material de referencia, quedando con ello demostrada la utilidad de los aditivos químicos.

Igualmente a los 28 días, la resistencia a compresión del hormigón con el "filler" + el aditivo químico era del 20 al $30 \%$ mayor que la del hormigón de referencia. Por otra parte, dicho valor era del 1 al $2 \%$ menor que el observado en las probetas con el aditivo químico sólo. Sin embargo, a los 60 días los hormigones con "filler" + aditivo químico mostraron valores de resistencia mucho mayores que los demás. Puede afirmarse que con el paso de los años la resistencia a compresión uniaxial del hormigón sería mayor en los hormigones con "filler".

La densidad del hormigón con "filler" también era mayor, debido a que éste rellenaba los huecos dentro de la matriz.

Tabla 4. Resultados de los ensayos practicados en el hormigón fresco y endurecido

\begin{tabular}{|c|c|c|c|c|c|c|c|c|c|c|}
\hline \multirow{2}{*}{$\begin{array}{l}\text { Cantidad } \\
\text { de } \\
\text { cemento } \\
(\mathbf{k g})\end{array}$} & \multirow{2}{*}{$\begin{array}{c}\text { Agua } \\
\text { de } \\
\text { amasado } \\
(\mathrm{kg})\end{array}$} & \multirow{2}{*}{$\begin{array}{c}\text { Aditivo } \\
\text { químico } \\
(\mathrm{kg})\end{array}$} & \multirow[t]{2}{*}{$\begin{array}{c}\text { "Filler" } \\
\text { (kg) }\end{array}$} & \multirow{2}{*}{$\begin{array}{l}\text { Asiento } \\
\text { en el } \\
\text { cono de } \\
\text { Abrams } \\
(\mathrm{cm})\end{array}$} & \multirow{2}{*}{$\begin{array}{c}\text { Contenido } \\
\text { de aire } \\
(\%)\end{array}$} & \multirow{2}{*}{$\begin{array}{c}\text { Peso por } \\
\text { unidad } \\
\text { de } \\
\text { volumen } \\
\left(\mathrm{kg} / \mathrm{cm}^{3}\right)\end{array}$} & \multicolumn{4}{|c|}{$\begin{array}{c}\text { Resultados de la resistencia } \\
\text { a compresión (MPa) }\end{array}$} \\
\hline & & & & & & & 3 días & 7 días & 28 días & 60 días \\
\hline 375 & 236 & 0 & 0 & 17 & 0,7 & 2.317 & 23,7 & 34,8 & 43,9 & 45,2 \\
\hline 375 & 189 & 5.625 & - & 16 & 2,1 & 2.364 & 35,5 & 45,4 & 57,6 & 58,8 \\
\hline 375 & 191 & 5.625 & 18,75 & 17 & 1,9 & 2.384 & 35,5 & 45,7 & 58,5 & 60,2 \\
\hline 400 & 226 & - & - & 18 & 0,9 & 2.331 & 27,9 & 41,0 & 49,9 & 51,4 \\
\hline 400 & 180 & 6.000 & - & 17 & 2,7 & 2.366 & 40,8 & 56,5 & 62,1 & 63,3 \\
\hline 400 & 190 & 6.000 & 20,00 & 16 & 2,2 & 2.383 & 37,9 & 55,1 & 62,8 & 63,8 \\
\hline 425 & 231 & - & - & 16 & 1,0 & 2.323 & 33,6 & 42,5 & 50,6 & 52,1 \\
\hline 425 & 194 & 6.400 & - & 16 & 2,2 & 2.359 & 48,2 & 55,9 & 64,2 & 65,4 \\
\hline 425 & 199 & 6.400 & 21,25 & 16 & 1,8 & 2.384 & 43,5 & 53,7 & 63,3 & 66,2 \\
\hline
\end{tabular}

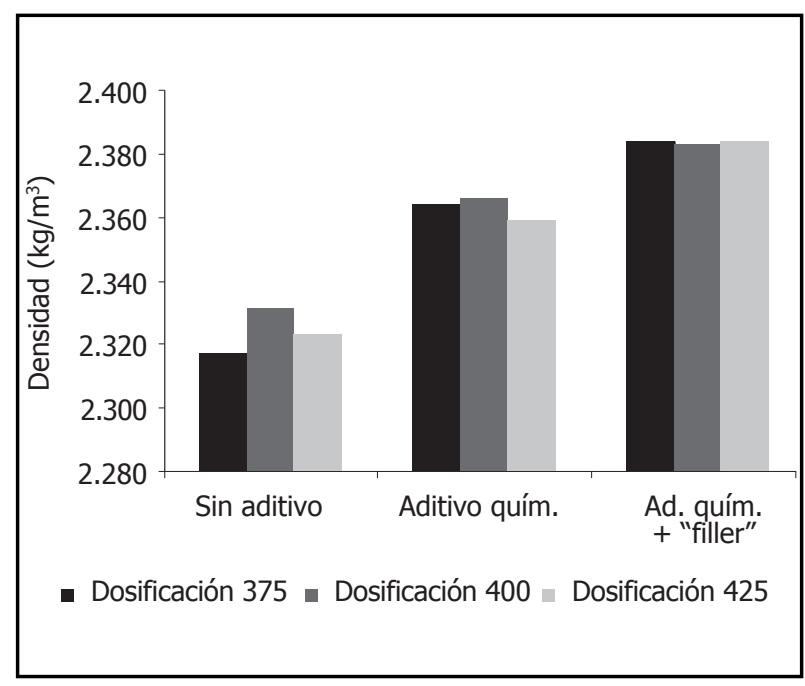

Figura 1. Peso por unidad de volumen del hormigón fresco.

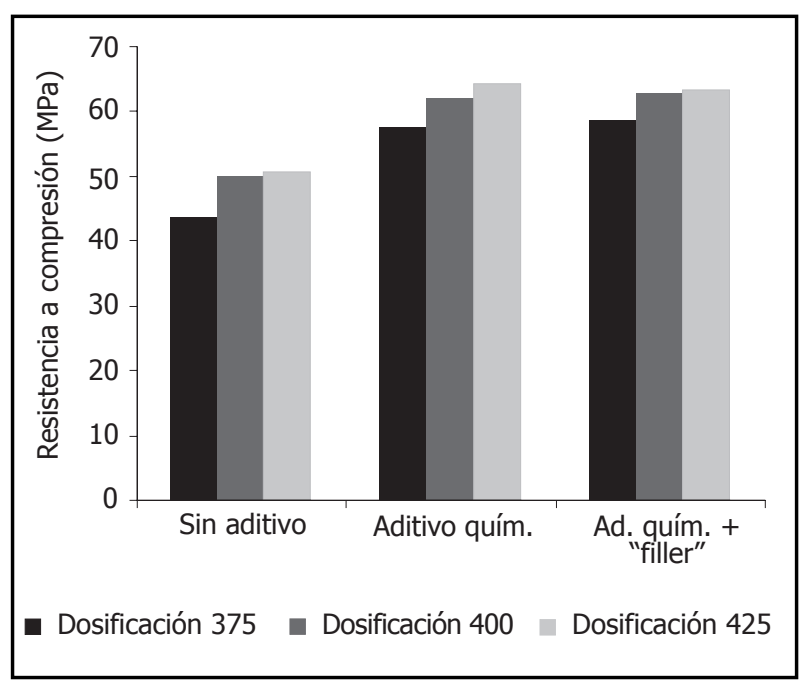

Figura 2. Resistencia a la compresión del hormigón endurecido a los 28 días. 


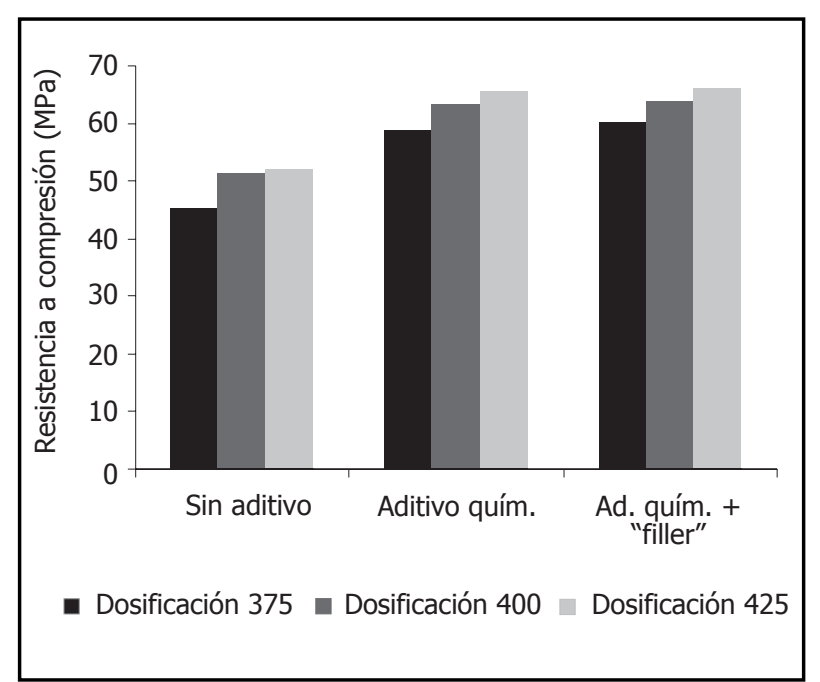

Figura 3. Resistencia a la compresión del hormigón endurecido a los 60 días.

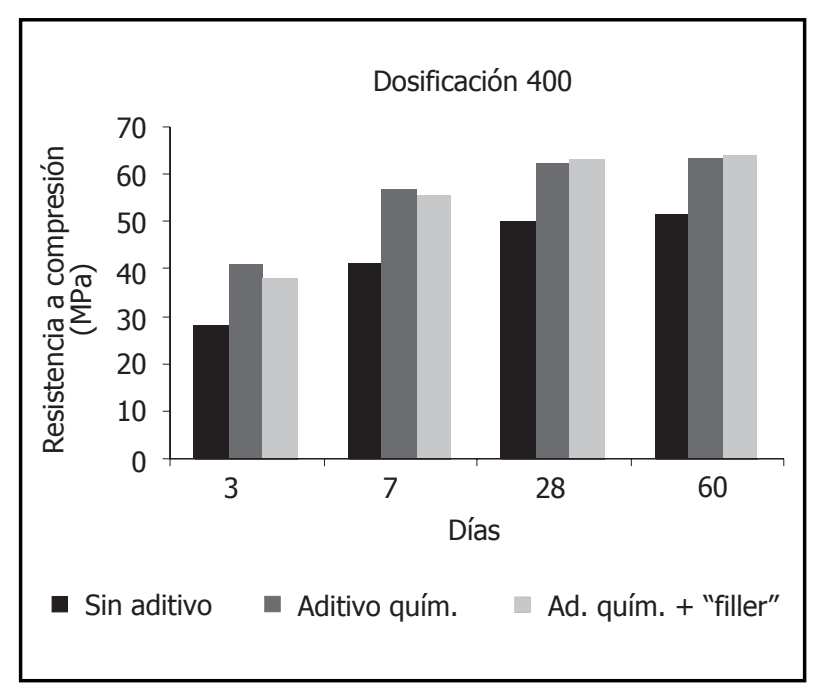

Figura 5. Resistencia a la compresión del hormigón endurecido con dosificación de cemento 400 .

La demanda de agua para alcanzar el valor establecido del asiento en el cono de Abrams era mayor en las muestras con "filler" + aditivo químico que en las que sólo llevaban el aditivo químico. Esto se justifica por la mayor superficie del "filler", que precisa por tanto de una mayor cantidad de agua para llegar a la saturación. Por otra parte, tanto la serie de hormigón con el aditivo químico como la que contenía el aditivo, además del "filler", tenían una demanda de agua menor, por el efecto del aditivo químico, que la serie de referencia.

En el presente trabajo, la dosis del "filler" era del 5\% de la del cemento. En trabajos futuros, deberán estudiarse otros valores del "filler", mayores y menores, a fin de determinar la proporción óptima.

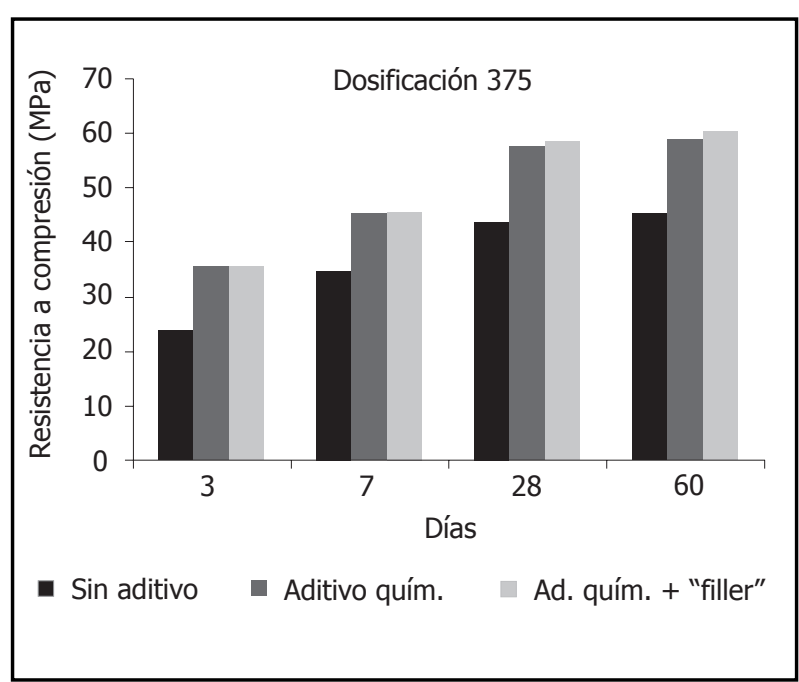

Figura 4. Resistencia a la compresión del hormigón endurecido con dosificación de cemento 375.

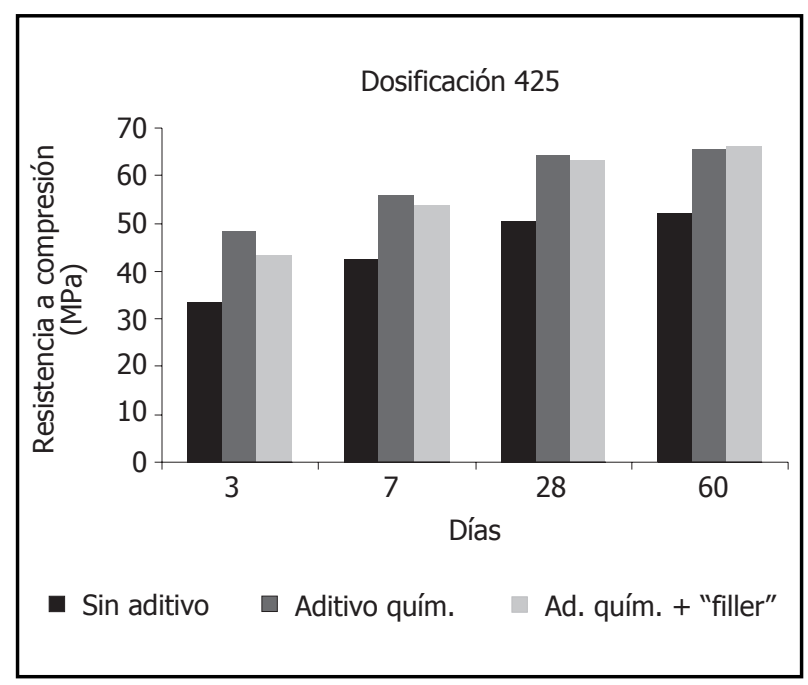

Figura 6. Resistencia a la compresión del hormigón endurecido con dosificación de cemento 425 .

Además, el efecto de otros residuos industriales como la ceniza volante, el humo de sílice y la escoria de altos hornos sobre las propiedades del hormigón debería evaluarse.

\section{CONCLUSIÓN}

En los últimos años, los niveles de contaminación ambiental en todo el mundo, pero especialmente en los países en desarrollo, han aumentado de forma alarmante. El "filler" que recogen los filtros de las canteras podría emplearse en el sector de la construcción, favoreciendo al sector en el aspecto económico y al medio ambiente mediante la disminución de la contaminación. 
Los estudios experimentales han demostrado la conveniencia, en la fabricación del hormigón, de sustituir el cemento por "fillers" con actividad puzolánica, como son la ceniza volante, el humo de sílice y las escorias de altos hornos, a fin de reducir la contaminación ambiental. En el presente trabajo, se ha sustituido el 5\% del cemento por "filler". En el hormigón con un contenido de "filler" del 5\%, la resistencia a compresión era un $9 \%$ mayor que en el hormigón al que se había agregado el superplastificante sólo, y de un 25 a un 33\% mayor que en el hormigón de referencia. En presencia del "filler", el contenido de aire disminuyó en un $10 \%$ en el hormigón con una dosificación de cemento de $375 \mathrm{~kg} / \mathrm{m}^{3}$, y en un $18 \%$ en las otros dos series de probetas, con respecto a los mismos hormigones con superplastificante sólo. Con esta configuración, el hormigón es más denso e impermeable, por lo que aumenta su resistencia a la compresión. Por consiguiente, se puede concluir que la utilización del "filler" en cementos y hormigones es muy beneficiosa.

\section{BIBLIOGRAFÍA}

(1) Öztekin, E., Sümer, M., et al.: "Some Compared Properties of Fly Ash and Filler Concretes", Industrial Waste Seminar, Ankara, 2328 November 1993.

(2) Akman, S. M.: "Materials of Construction", Istanbul Technical University Lecture Notes, Istanbul, 1998.

(3) Topcu, B. I., Uğurlu, A.: "Effect of the use of mineral filler on the properties of concrete", Cem. Concr. Res. Journal accepted 2 January 2003.

(4) Canpolat, F., Yılmaz, K., Köse, M., Sümer, M., Yurdasev, M. A.: "Use of Zeolit, Coal Bottom ash and Fly-ash Replacement Materials in cement Production", Cem. Concr. Res. Journal accepted 7 February 2003.

(5) Postacıoğlu, B.: . Istanbul, Istanbul Technical University Technical Books Publishing House, 1987.

(6) Cark, A. I.: "An Investigation on the Effects of Some Properties of Concrete", MSc Thesis, Famagusta, Eastern Mediterranean University, 1995. 